\title{
Differentiation between Graves' disease and painless thyroiditis by diffusion-weighted imaging, thyroid iodine uptake, thyroid scintigraphy and serum parameters
}

\author{
ZHAOWEI MENG ${ }^{1}$, GUIZHI ZHANG ${ }^{1}$, HAORAN SUN ${ }^{2}$, JIAN TAN ${ }^{1}$, CHUNSHUN YU $^{2}$, WEIJUN TIAN ${ }^{3}$, \\ WEIDONG $\mathrm{LI}^{3}$, ZHIQIANG YANG ${ }^{3}$, MEI ZHU $^{4}$, QING HE ${ }^{4}$, YUJIE ZHANG ${ }^{5}$ and SHUGAO HAN ${ }^{1,6}$ \\ Departments of ${ }^{1}$ Nuclear Medicine, ${ }^{2}$ Radiology, ${ }^{3}$ General Surgery, ${ }^{4}$ Endocrinology and ${ }^{5}$ Pathology, \\ Tianjin Medical University General Hospital, Tianjin 300052; ${ }^{6}$ Department of Radiology, \\ Second Affiliated Hospital of Zhejiang Medical University, Hangzhou, Zhejiang 310000, P.R. China
}

Received July 30, 2014; Accepted April 13, 2015

DOI: $10.3892 /$ etm.2015.2430

\begin{abstract}
The aim of the present study was to assess the apparent diffusion coefficient (ADC) in diffusion-weighted imaging (DWI), thyroid radioactive iodine uptake (RAIU), thyroid scintigraphy and thyrotropin receptor antibody (TRAb) levels in the differential diagnosis between Graves' disease (GD) and painless thyroiditis (PT). A total of 102 patients with GD and 37 patients with PT were enrolled in the study. DWI was obtained with a 3.0-T magnetic resonance scanner, and ADC values were calculated. RAIU and thyroid scintigraphy were performed. Tissue samples were obtained from patients with GD (6 cases) following thyroidectomy, and from patients with PT (2 cases) following biopsy. Receiver operating characteristic (ROC) curves were drawn, optimal cut-off values were selected, and the sensitivity, specificity, accuracy, positive predictive value (PPV) and negative predictive value (NPV) were assessed. It was found that the ADC, TRAb and RAIU were significantly higher in GD than in $\mathrm{PT}(\mathrm{P}<0.05)$. ROC curves showed areas under the curves for RAIU, ADC and TRAb that were $>0.900$. RAIU was the reference method. Sensitivity, specificity, accuracy, PPV and NPV were 96.078, 91.892, 95.000, 97.059 and 89.474\% for ADC, and 88.235, $75.676,84.892,90.909$ and $70.000 \%$ for $\mathrm{TRAb}$, after the optimal thresholds of $1.837 \times 10^{-3} \mathrm{~mm}^{2} / \mathrm{sec}$ and $1.350 \mathrm{IU} / \mathrm{ml}$ were determined respectively. Histopathology showed that tissue cellularity in PT was much higher than in GD due to
\end{abstract}

Correspondence to: Dr Zhaowei Meng or Dr Jian Tan, Department of Nuclear Medicine, Tianjin Medical University General Hospital, 154 Anshan Road, Heping, Tianjin 300052, P.R. China

E-mail: jamesmencius@163.com

E-mail: tanpost@163.com

Key words: Graves' disease, painless thy roiditis, diffusion-weighted magnetic resonance imaging, apparent diffusion coefficient, thyroid radioactive iodine uptake, thyroid scintigraphy, thyrotropin receptor antibody massive lymphocytic infiltration. The results of the present study indicate that RAIU, ADC and TRAb are of diagnostic value for differentiating between GD and PT. DWI has great potential for thyroid pathophysiological imaging because it reflects differences in tissue cellularity between GD and PT.

\section{Introduction}

Differentiation between stimulation-induced thyrotoxicosis and destruction-induced thyrotoxicosis is important (1). The former refers to Graves' disease (GD), while the latter includes subacute thyroiditis, painless thyroiditis (PT, also called silent thyroiditis) and postpartum thyroiditis. Therapy for the two entities is completely different. Antithyroid drugs (ATDs), radioactive iodine $131\left({ }^{131} \mathrm{I}\right)$ and thyroidectomy are the treatments for GD, whereas destruction-induced thyrotoxicosis can be managed conservatively. Treating PT as if it were GD would be completely inappropriate (1). The diagnosis of subacute thyroiditis is not difficult because is caused by viral infection and is characterized by fever and thyroid pain. However, PT is easily overlooked or misdiagnosed and perhaps mistreated since its symptoms resemble those of early onset or recurrence of GD. It is often problematic to discriminate GD from PT unless radioactive iodine uptake (RAIU) is determined, because occasionally GD and PT are associated with each other. PT may develop following the complete remission of GD, and PT can be followed by GD (2-10).

Although RAIU is the most reliable method to differentiate GD from PT (11-14), it is contraindicated when patients are lactating, and not all clinics are well equipped to perform RAIU. Therefore, a number of studies have been undertaken to search for simple and practical parameters that might allow differentiation between the two entities. Thyrotropin receptor antibody (TRAb) assessment has been shown to be a useful marker to make a distinction between the two diseases (2-3,15-16). TRAb is also able to differentiate relapse of GD from development of PT in patients who appear to be in remission following ATD treatment for GD (2). However, $5-10 \%$ of GD patients are negative for TRAb, and some PT patients are positive (17-19). Amino et al demonstrated that 
the serum triiodothyronine (T3)/thyroxine (T4) ratio (20) or free triiodothyronine (FT3)/free thyroxine(FT4) ratio (21) was useful for differentiating PT from GD. However, these observations have not been confirmed by other groups.

Several methods of thyroid imaging can be used for differential diagnosis. Thyroid scintigraphy using ${ }^{99 \mathrm{~m}} \mathrm{Tc}$-pertechnetate has been well established for use in the assessment of thyroid uptake ability. Although ${ }^{99 \mathrm{~m} T c-p e r t e c h n e t a t e ~ d o e s ~ n o t ~ u n d e r g o ~}$ organification in the thyroid, the pertechnetate ion is transported into the thyroid by the sodium/iodide symporter. Thus, thyroid scintigraphy embodies and enables the visualization of thyroid RAIU (12). Thyroid volume and blood flow quantitative measurement by ultrasonography has been shown to be effective for differential diagnosis (22). Diffusion-weighted magnetic resonance imaging (DWI) of the thyroid with the assessment of an apparent diffusion coefficient (ADC) value is a relatively new topic in thyroid imaging studies. There appears to be only one study in which DWI has been used to differentiate between GD and thyroiditis. Tezuka et al (23) demonstrated that the ADC values of patients with GD were significantly higher than those of patients with subacute thyroiditis and Hashimoto thyroiditis. However, to the best of our knowledge, no prior study has investigated whether DWI is useful for discriminating between GD and PT. Furthermore, the total number of cases in the study by Tezuka et al was only 34 , and the results of the study require verification.

In this study, the aim was to systematically evaluate the ADC value in DWI for the differentiation between GD and PT, and to compare it with RAIU (the reference method), thyroid scintigraphy, TRAb and other serum indices. Parameters were compiled and statistically analyzed to determine sensitivity, specificity, accuracy, positive predictive value (PPV) and negative predictive value (NPV) for differentiation diagnosis. Tissue histopathology of GD and PT was also investigated.

\section{Materials and methods}

Patients. From August 2010 until August 2013, a series of 102 patients with GD and 37 patients with PT were consecutively enrolled in this prospective study. The Institutional Review Board of Tianjin Medical University General Hospital (Tianjin, China) approved the ethical and methodological aspects of this investigation. All participants provided their written informed consent to participate in this study. Diagnosis was made according to the generally recognized guidelines (1), with consensus. In brief, GD was diagnosed on the basis of clinical findings and laboratory tests showing high values of free thyroid hormone, low levels of thyroid-stimulating hormone (TSH), high RAIU and/or increased TRAb activity. PT was diagnosed by increased free thyroid hormone levels and low TSH levels for $<3$ months, low RAIU and/or later development of transient hypothyroidism.

Evaluation of serum parameters. Assays to determine the levels of FT3 (reference, 3.50-6.50 pmol/1; maximum, $30.80 \mathrm{pmol} / \mathrm{l}$ ), FT4 (reference, $11.50-23.50 \mathrm{pmol} / \mathrm{l}$; maximum, $154.80 \mathrm{pmol} / \mathrm{l}$ ) and TSH (reference, 0.30-5.00 $\mu \mathrm{IU} / \mathrm{ml}$ ) were performed on a fully automated ADVIA Centaur analyzer (Siemens Healthcare Diagnostics, Tarrytown, NY, USA). These assays were based on a chemiluminescent reaction principle.
Thyroid globulin antibody (TgAb; reference, $0-40.00 \mathrm{IU} / \mathrm{ml}$; maximum, 3,000.00 IU/ml) and thyroid peroxidase antibody (TpoAb; reference, 0-35.00 IU/ml; maximum 1,000.00 IU/ml) were also assessed by chemiluminescent reaction on a fully automated IMMULITE 2000 analyzer (Siemens Healthcare Diagnostics, Los Angeles, CA, USA). TRAb (reference, 0-1.50 IU/1; maximum, $40.00 \mathrm{IU} / 1$ ) was determined by a competitive enzyme immunoassay (Medizym T.R.A., Medipan GmbH, Berlin, Germany).

$D W I$ and $A D C$. Magnetic resonance (MR) images were obtained with a superconducting 3.0-T MR imaging unit (Signa HDx; GE Healthcare, Milwaukee, WI, USA) using an anterior neck array coil. The neck array coil was carefully placed in order to position the thyroid gland in the center of the field of view. T1-weighted images were acquired using the following parameters: repetition time (TR), $780 \mathrm{msec}$; echo time (TE), $11 \mathrm{msec}$; slice thickness, $5.0 \mathrm{~mm}$; intersection gap, $1.0 \mathrm{~mm}$; matrix, 320x224; field of view (FOV), 240x240 $\mathrm{mm}^{2}$; and echo train length, 3. T2-weighted images were obtained by using the following parameters: TR, 5,000 msec; TE, $102 \mathrm{msec}$; slice thickness, $5.0 \mathrm{~mm}$; intersection gap, $1.0 \mathrm{~mm}$; matrix, 288x256; FOV, 240x240 $\mathrm{mm}^{2}$; and echo train length, 18. DWI was conducted with the following parameters: TR, 5,000 msec; TE, $75 \mathrm{msec}$; slice thickness, $5.0 \mathrm{~mm}$; intersection gap, $1.0 \mathrm{~mm}$; matrix, 128x128; FOV, 240x240 mm²; and echo train length, 20. Imaging was performed with $b$ values of 0 and $1,000 \mathrm{sec} / \mathrm{mm}^{2}$. Afterwards, an ADC map was constructed and ADC values were automatically calculated in units of $\mathrm{x} 10^{-3} \mathrm{~mm}^{2} / \mathrm{sec}$ on an ADW 4.3 workstation (GE Healthcare, Waukesha, WI, USA). The MR imaging signal intensities of the thyroid gland were measured with an electronic cursor to define the region of interest (ROI). On each patient, ROIs were drawn around bilateral thyroid parenchyma at the level of the upper pole, central portion and the lower pole, while avoiding artifacts from focal lesions, vascular motion, chemical shift or magnetic susceptibility. The final ADC per subject that used for statistical analysis was the average of the above ADC values in one patient.

RAIU and thyroid scintigraphy. As reference methods, RAIU and thyroid scintigraphy were performed as previously described $(24,25)$. Briefly, the thyroid RAIU protocol was performed as follows: Radioactivities of the thyroid as well as the decayed tracer source were measured at $24 \mathrm{~h}$ after oral intake of the tracer dose $\left(74 \mathrm{kBq}\right.$ of $\left.{ }^{131} \mathrm{I}\right)$ using a multifunctional nuclear medicine instrument (MN-6300XT; University of Science and Technology of China, Hefei, China). The RAIU was calculated using the formula: RAIU $(\%)=[$ (radioactivity of the thyroid - background)/(radioactivity of the decayed tracer source - background)] x 100. The thyroid scintigraphy protocol was implemented as follows: $30 \mathrm{~min}$ after the injection of $185 \mathrm{MBq}{ }^{99 \mathrm{~m}} \mathrm{Tc}$-pertechnetate, acquisition was performed using a high-resolution low-energy parallel-hole collimator equipped dual-detector scanner (Discovery VH; GE Healthcare, Milwaukee, WI, USA). Thyroid scintigraphy was performed subsequent to RAIU measurement.

Treatments and tissue sampling. Treatments were determined according to the generally accepted guidelines (1). GD was 
Table I. Comparisons among the factors of the two groups of patients in the study.

\begin{tabular}{lccc}
\hline Factor & Graves' disease $(\mathrm{n}=102)$ & Painless thyroiditis $(\mathrm{n}=37)^{\text {F value }(\mathrm{P}-\mathrm{value})^{\mathrm{a}}}$ \\
\hline Age & $37.971 \pm 13.856$ & $35.054 \pm 12.326$ & $2.083(0.151)$ \\
ADC & $2.212 \pm 0.209$ & $1.508 \pm 0.318$ & $5.979(0.016)$ \\
FT3 & $15.555 \pm 6.507$ & $11.336 \pm 4.111$ & $4.491(0.036)$ \\
FT4 & $68.868 \pm 31.608$ & $54.598 \pm 22.049$ & $1.479(0.226)$ \\
TSH & $0.007 \pm 0.008$ & $0.008 \pm 0.016$ & $0.884(0.349)$ \\
TRAb & $9.378 \pm 6.877$ & $1.123 \pm 1.472$ & $42.951(<0.001)$ \\
TgAb & $317.001 \pm 718.324$ & $415.379 \pm 630.150$ & $0.006(0.940)$ \\
TpoAb & $444.047 \pm 389.482$ & $464.341 \pm 337.990$ & $3.625(0.059)$ \\
RAIU & $66.358 \pm 11.799$ & $3.678 \pm 4.075$ & $25.466(<0.001)$ \\
FT3/FT4 & $0.237 \pm 0.045$ & $0.219 \pm 0.051$ & $1.649(0.201)$ \\
\hline
\end{tabular}

ADC, apparent diffusion coefficient; FT3, free triiodothyronine; FT4, free thyroxine; TSH, thyroid-stimulating hormone; TRAb, thyrotropin receptor antibody; $\mathrm{TgAb}$, thyroid globulin antibody; $\mathrm{TpoAb}$, thyroid peroxidase antibody; RAIU, radioactive iodine uptake. ${ }^{\mathrm{a} A n a l y z e d}$ by least significant independent samples t-test.

managed by one of the following therapies: ATD, ${ }^{131} \mathrm{I}$ or thyroidectomy. Decisions about the therapy were made at a consultation meeting among endocrinologists, nuclear medicine physicians and surgeons. PT was given symptomatic treatments, for example, $\beta$-adrenergic blockers, nonsteroidal anti-inflammatory agents, and sometimes corticosteroids to ameliorate symptoms. Tissue samples were obtained from patients with GD following thyroidectomy, and from patients with PT following biopsy. Tissue samples were fixed in neutral buffered formalin ( $\mathrm{pH} 7.4$ ), embedded in paraffin, and sliced into $\sim 4-\mu \mathrm{m}$ sections by a routine procedure. Sections were stained with hematoxylin and eosin in order to examine the general histology. Images were acquired and observed under a microscope (BX51; Olympus, Tokyo, Japan).

Statistical analysis. All data are presented as mean \pm standard deviation. Statistics were performed with SPSS software, version 17.0 (SPSS, Inc., Chicago, IL, USA). Differences of indices between two groups were analyzed by independent samples t-test. The Pearson $\chi^{2}$ test was used to check whether gender had a significant effect on inter-group differences. Pearson bivariate correlation was performed among the variables. Receiver operating characteristic (ROC) curves were drawn and diagnostic efficacies were determined by comparing the areas under the curves. Then, optimal cut-off values were selected, and the sensitivity, specificity, diagnostic accuracy, PPV and NPV of various factors for differential diagnosis were assessed, respectively. P-values not exceeding 0.05 were considered statistically significant.

\section{Results}

Comparisons of clinical indices between different groups of patients. For the GD group, there were 31 males (age range, 16-59 years) and 71 females (age range, 19-65 years). For the PT group, there were 10 males (age range, $21-60$ years) and 27 females (age range, 21-61 years). Gender did not have any substantial impact on the differential diagnosis of the two diseases, with a Pearson $\chi^{2}$ value of $0.148(\mathrm{P}=0.701)$.
Table II. Pearson bivariate correlation.

\begin{tabular}{lccc}
\hline Factor & ADC & TRAb & RAIU \\
\hline Age & 0.009 & -0.055 & 0.079 \\
ADC & - & $0.777^{\mathrm{a}}$ & $0.902^{\mathrm{a}}$ \\
FT3 & $0.524^{\mathrm{a}}$ & $0.730^{\mathrm{a}}$ & $0.479^{\mathrm{a}}$ \\
FT4 & $0.436^{\mathrm{a}}$ & $0.652^{\mathrm{a}}$ & $0.401^{\mathrm{a}}$ \\
TSH & -0.060 & -0.055 & -0.075 \\
TRAb & $0.777^{\mathrm{a}}$ & - & $0.731^{\mathrm{a}}$ \\
TgAb & -0.043 & -0.037 & -0.095 \\
TpoAb & 0.015 & $-0.252^{\mathrm{a}}$ & -0.082 \\
RAIU & $0.902^{\mathrm{a}}$ & $0.731^{\mathrm{a}}$ & - \\
FT3/FT4 & 0.031 & -0.085 & 0.051 \\
\hline
\end{tabular}

ADC, apparent diffusion coefficient; FT3, free triiodothyronine; FT4, free thyroxine; TSH, thyroid-stimulating hormone; TRAb, thyrotropin receptor antibody; $\mathrm{TgAb}$, thyroid globulin antibody; $\mathrm{TpoAb}$, thyroid peroxidase antibody; RAIU, radioactive iodine uptake. ${ }^{\mathrm{a}} \mathrm{P}<0.01$.

Clinical indices of the two groups of patients are listed and compared in Table I. The ADC, FT3 and TRAb levels and RAIU were significantly higher in the GD group than in the PT group $(\mathrm{P}<0.05)$. However, no significant differences were identified among the other parameters, namely age, FT4, TSH, TgAb, TpoAb and FT3/FT4 (P>0.05).

Pearson bivariate correlations were carried out. Table II demonstrates that for ADC, the three highest correlation coefficients were with RAIU, TRAb and FT3. For TRAb they were ADC, RAIU and FT3, and for RAIU they were ADC, TRAb and FT3. Correlation coefficients among RAIU, ADC, TRAb were $>0.700$. Therefore, RAIU, ADC and TRAb were closely and positively correlated with each other.

Imaging performance. The MR images of an index case of GD and an index case of PT are presented in Fig. 1. Signal intensities of T1-weighted images (A,D) and T2-weighted 
A

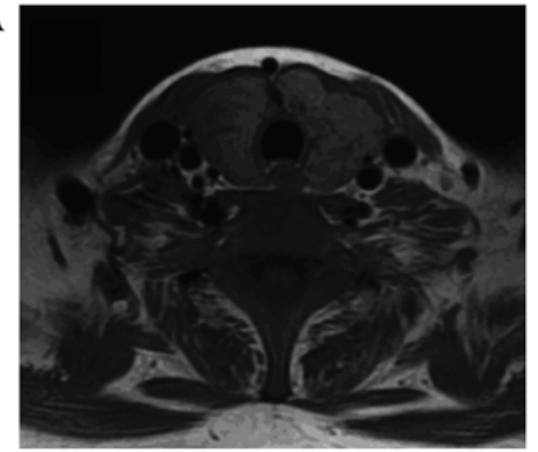

C

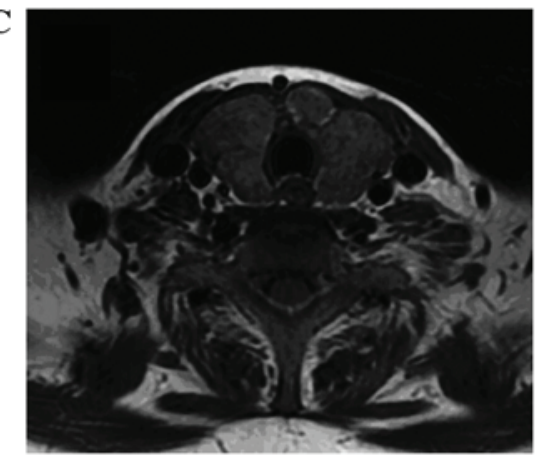

$\mathbf{E}$

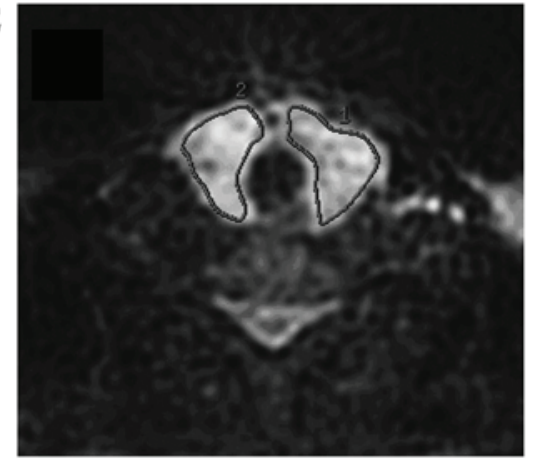

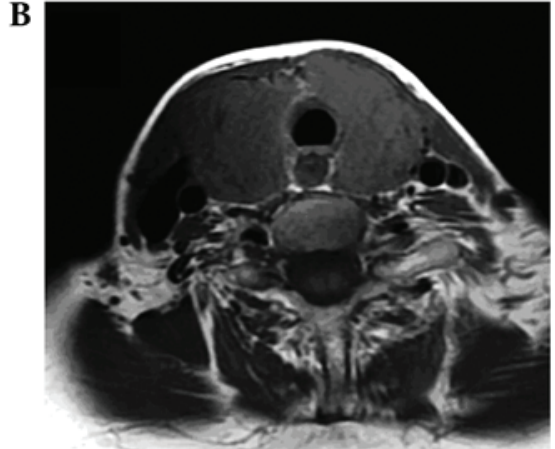

D
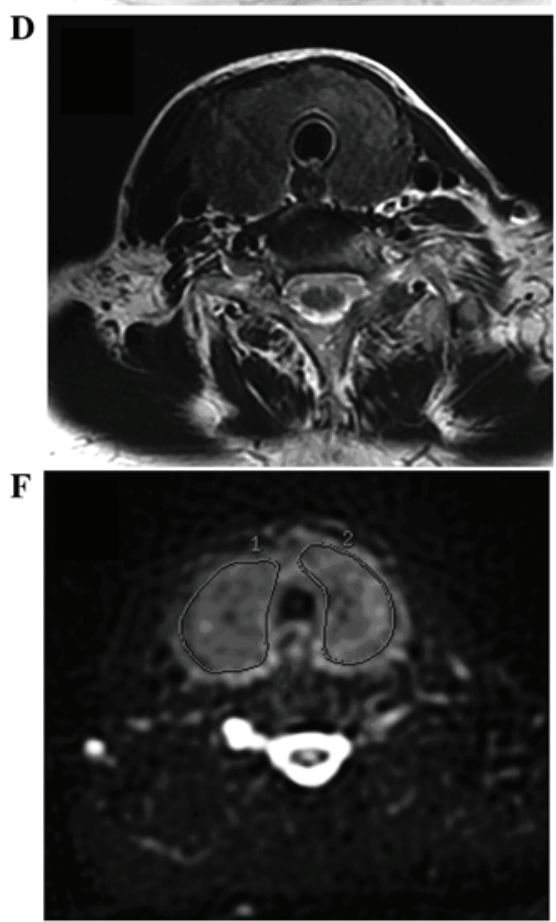

Figure 1. Diffusion-weighted magnetic resonance imaging with the assessment of apparent diffusion coefficient. Diffusion-weighted magnetic resonance imaging was performed as described in Materials and methods. (A-C) An index case of Graves' disease and (D-F) an index case of painless thyroiditis are presented. T1-weighted images (A and D), T2-weighted images (B and E) and apparent diffusion coefficient maps from diffusion-weighted imaging (C and F) are presented. The region of interest is drawn on the diffusion-weighted images (C and F).

A

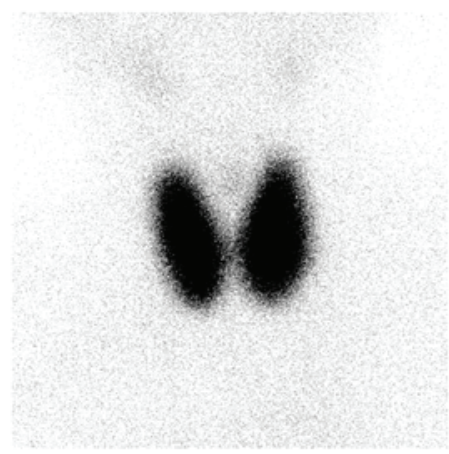

B

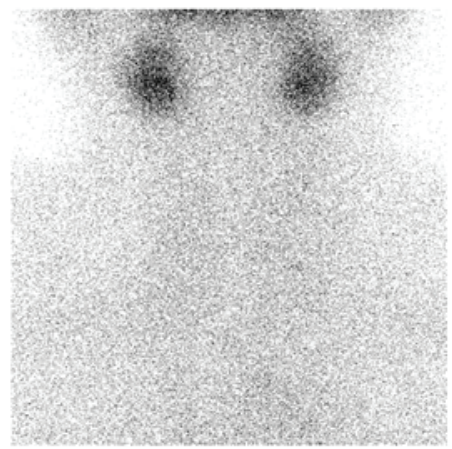

Figure 2. Thyroid scintigraphy of the participants. Thyroid scintigraphy was performed as described in materials and methods. An index case of Graves' disease (A) and an index case of painless thyroiditis were presented (B).

images $(\mathrm{B}, \mathrm{E})$ were not different, while ADC maps from DWI displayed significantly higher signal intensity in GD than in $\mathrm{PT}(\mathrm{C}, \mathrm{F})$. Thyroid scintigraphy results of an index case of GD and an index case of PT are presented in Fig. 2. The uptake of radionuclide ${ }^{99 \mathrm{~m}} \mathrm{Tc}-$ pertechnetate was significantly higher in the patient with GD (Fig. 2A) than in the patient with PT (Fig. 2B), which visually reflected the difference in RAIU between the two diseases. 
Table III. Az values in receiver operating characteristic curve among factors.

\begin{tabular}{lcr}
\hline Factor & $\mathrm{Az}$ & P-value \\
\hline Age & 0.554 & 0.331 \\
ADC & 0.980 & $<0.001$ \\
FT3 & 0.706 & $<0.001$ \\
FT4 & 0.620 & 0.030 \\
TSH & 0.479 & 0.701 \\
TRAb & 0.925 & $<0.001$ \\
TgAb & 0.348 & 0.006 \\
TpoAb & 0.484 & 0.780 \\
RAIU & 1.000 & $<0.001$ \\
FT3/FT4 & 0.603 & 0.064
\end{tabular}

$\mathrm{Az}$, area under the curve; ADC, apparent diffusion coefficient; FT3, free triiodothyronine; FT4, free thyroxine; TSH, thyroid-stimulating hormone; TRAb, thyrotropin receptor antibody; $\mathrm{TgAb}$, thyroid globulin antibody; TpoAb, thyroid peroxidase antibody; RAIU, radioactive iodine uptake.

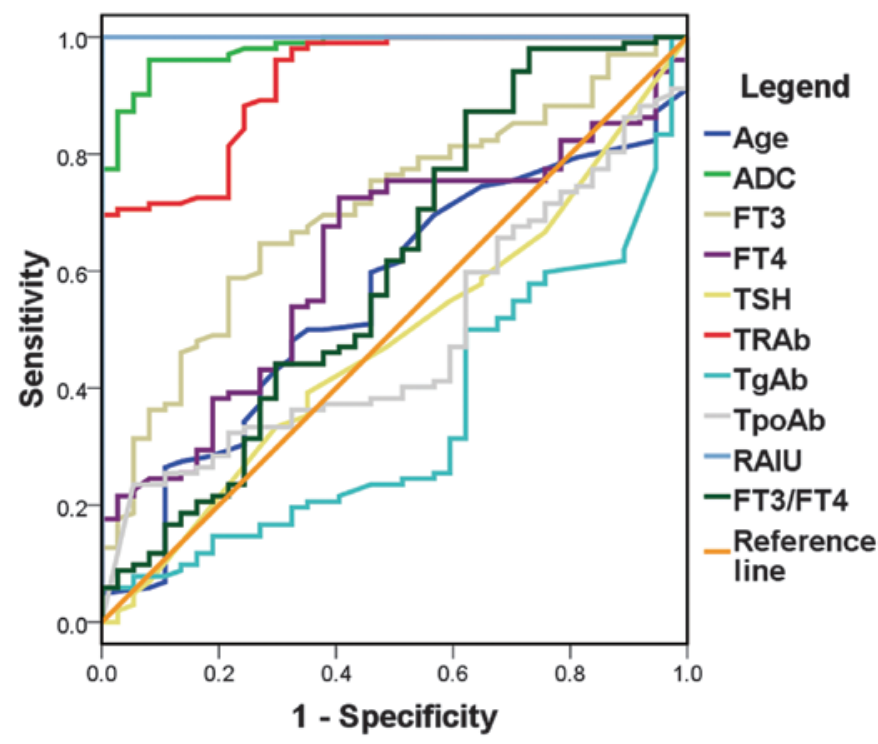

Figure 3. Receiver operating characteristic curves. Receiver operating characteristic curves were drawn to assess the diagnostic capabilities of the following factors: Age, ADC (apparent diffusion coefficient), FT3 (free triiodothyronine), FT4 (free thyroxine), TSH (thyroid-stimulating hormone), $\mathrm{TRAb}$ (thyrotropin receptor antibody), $\mathrm{Tg} \mathrm{Ab}$ (thyroid globulin antibody), TpoAb (thyroid peroxidase antibody), RAIU (radioactive iodine uptake) and FT3/FT4 ratio.

Diagnostic efficacy of various indices. ROC curves were drawn and are shown in Fig. 3. Diagnostic capabilities, indicated by area under the curve $(\mathrm{Az})$ value, decreased in the following order: RAIU $>$ ADC $>$ TRAb $>$ FT3 $>$ FT4 $>$ FT3/FT4 $>$ age $>\mathrm{TSH}>\mathrm{TpoAb}>\mathrm{TgAb}$ (Table III). There were three factors (RAIU, ADC and TRAb) that had an Az $>0.900$, which indicated that they had excellent diagnostic efficacy. The cut-off value, sensitivity, specificity, diagnostic accuracy, PPV and NPV of ADC, TRAb and RAIU are listed in Table IV. RAIU was used as the reference method. When the optimal threshold

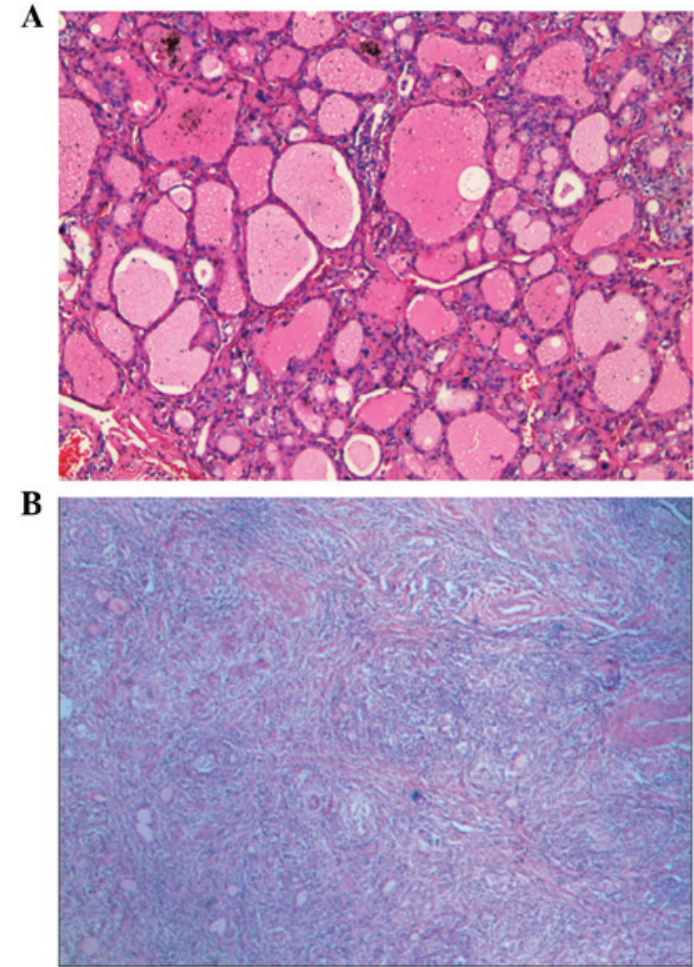

Figure 4. Histopathological examination. Tissue samples were obtained, sliced and stained for general histology as described in Materials and methods. Representative images of (A) Graves' disease and (B) painless thyroiditis were acquired and observed under a microscope (hematoxylin and eosin staining; magnification, x100).

of $24.500 \%$ was set, the sensitivity, specificity, accuracy, PPV and NPV were all $100 \%$. For ADC and TRAb, the optimal thresholds of $1.837 \times 10^{-3} \mathrm{~mm}^{2} / \mathrm{sec}$ and $1.350 \mathrm{IU} / \mathrm{ml}$ were determined. ADC had better diagnostic capability than TRAb, as the sensitivity, specificity, accuracy, PPV and NPV were all higher for the former index.

Therapeutic management and histopathological comparison. For the 102 patients with GD, treatment with ${ }^{131}$ I, ATD and thyroidectomy was given to 78, 18 and 6 patients respectively. All 37 patients with PT were managed with $\beta$-adrenergic blockers. In addition, nonsteroidal anti-inflammatory agents were prescribed to 21 patients and corticosteroids were administrated to 8 patients. Tissue samples were obtained from the 6 patients with GD who received thyroidectomy and 2 patients with recurrent PT who agreed to undergo biopsy, and were observed by microscopy.

For GD, the follicular epithelial cells were tall and more crowded than those of a normal thyroid gland. Some small papillae were formed, which projected into the follicular lumen and encroached on the colloid. The colloid within the follicular lumen was pale, with scalloped margins. Lymphoid infiltrates were present in the interstitium (Fig. 4A). For PT, the most prominent and specific histopathological feature was the massive lymphocytic infiltration with hyperplastic germinal centers within the thyroid parenchyma. Thyroid follicles were disrupted and collapsed (Fig. 4B). It was evident that the tissue cellularity in PT was much higher than that in GD. 
Table IV. Receiver operating characteristic curve-related data and diagnostic indices.

\begin{tabular}{lccr}
\hline Factor & ADC & TRAb & RAIU \\
\hline Cut-off value & $1.837 \times 10^{-3} \mathrm{~mm}^{2} / \mathrm{sec}$ & $1.350 \mathrm{IU} / \mathrm{ml}$ & 24.500 \\
Sensitivity (\%) & 96.078 & 88.235 & 100.000 \\
Specificity (\%) & 91.892 & 75.676 & 100.000 \\
Accuracy (\%) & 95.000 & 84.892 & 100.000 \\
PPV (\%) & 97.059 & 90.909 & 100.000 \\
NPV (\%) & 89.474 & 70.000 & 100.000 \\
\hline
\end{tabular}

NPV, negative predictive value; PPV, positive predictive value; RAIU, radioactive iodine uptake; ADC, apparent diffusion coefficient; TRAb, thyrotropin receptor antibody.

\section{Discussion}

GD and PT are two distinct clinical entities within the wide spectrum of autoimmune thyroid diseases. Thyrotoxicosis induced by PT is usually self-limited, and therefore only requires symptomatic treatment. Therapies intended for GD, in particular ATD, ${ }^{131} \mathrm{I}$ or thyroidectomy, are usually contraindicated for PT. Differential diagnosis of these two types of thyrotoxicosis has recently become an important clinical concern. However, differentiation is sometimes challenging on the basis of clinical findings only. First, PT is not rare. PT has a relatively high prevalence, accounting for 9-23\% of all thyrotoxicosis cases (26). PT often present with milder symptoms, so many patients with PT will often go to a general practitioner, rather than a specialized thyroid clinic. Therefore, the actual prevalence of PT is likely to be even higher than is recognized. Secondly, hyperthyroidism, ophthalmopathy, diffuse goiter and the peau d'orange appearance of pretibial dermopathy are the major manifestations of GD (27), although not all GD patients exhibit these signs. Without clear clinical manifestations, differentiation will be more difficult. Thirdly, these two diseases are often intertwined with each other. PT can often develop following the remission of GD, PT can also be followed by GD, and in rare cases simultaneous occurrence of the two can happen (10).

Generally, GD is characterized by persistent thyrotoxicosis associated with a hyperfunctional thyroid gland that gives rise to avid uptake of radioiodine. By contrast, PT is a clinical syndrome manifested by spontaneously resolving thyrotoxicosis associated with thyroidal destruction and marked suppression of thyroid iodine uptake. Pathologically, GD is characterized by diffuse hyperplasia and hypertrophy of follicular cells with retention of lobular architecture and prominent vascular congestion. Tall follicular cells with papillae are often observed. PT is a syndrome of thyrotoxicosis due to the release of preformed thyroid hormones from disrupted thyroid follicles. The diffuse infiltration of the thyroid by lymphocytes implies that it is also an autoimmune disorder $(28,29)$. RAIU testing or thyroid scintigraphy is of high diagnostic value for differentiating between GD and PT, and has been demonstrated to be reliable (11-14); however, RAIU and thyroid scintigraphy reflect only one type of change in the pathogenesis of the two diseases. In brief, enhanced thyrocyte uptake and processing of iodine in the former, and disruption of thyroid follicles and suppressed thyrocyte uptake in the latter. The more condense cellular structure of PT in comparison with that in GD, due to massive lymphocyte infiltration with hyperplastic germinal centers, is another hallmark histopathological difference, which has not been studied in terms of imaging so far.

DWI has a high sensitivity in the detection of changes in the microscopic cellular environment. In particular, DWI noninvasively probes the random microscopic motion of free water molecules (known as Brownian motion) in a defined voxel by means of the application of motion-probing gradients. The movement of water is affected by cell organization, density, microstructure, microcirculation and interaction with tissue compartments. DWI is quantified by measuring the ADC value in units of $\mathrm{mm}^{2} / \mathrm{sec}$, which defines the average area covered by a molecule per unit time. The ADC value can be calculated by assessing the signal attenuation that occurs in DWI performed at different b values. Usually, low ADC values indicate restricted diffusion (high cellular density), while high ADC values indicate more free diffusion (low tissue cellularity) (30-32).

DWI has a wide range of diagnostic applications. It has primarily been used clinically for brain disorders, particularly for the early detection of ischemic stroke or infarction (33-36). Numerous studies have put forward DWI as a new and valuable cancer imaging biomarker for detection, diagnosis, staging, detecting metastasis or relapse, and assessing treatment response (30-32,37-44). Studies have also demonstrated that, for malignancy imaging, the quantitative analysis of ADC and the standard uptake value (SUV) in fluorodeoxyglucose positron emission tomography (FDG PET)/CT are inversely correlated (37-42). ADC and SUV play complementary roles in the provision of functional information concerning cancer, which can now be successfully applied in head and neck cancers, after overcoming the motion artifacts from swallowing or respiration and susceptibility artifacts due to air/soft tissue/ bone interfaces (38-40). FDG PET/CT traces glucose metabolism, a nonspecific process essential for tumor growth. DWI provides information on the random motion of water molecules in tissues indicating cellularity, as well as intra-cellular and inter-cellular membranes. Tissue glucose metabolism and cellularity represent two different facets of tumor biology and pathophysiology. These successful applications of DWI prompted the investigation in the present study of whether DWI can be used for differential diagnosis of GD and PT.

To the best of our knowledge, the current investigation is the first to apply DWI to discriminate GD from PT, and make correlations and comparisons among ADC, RAIU and serum indices. The findings of the present study demonstrate the definite confirmative diagnostic ability of RAIU with $100 \%$ accuracy. It was also found that the RAIU values are positively and closely associated with those of ADC and TRAb. The performance of ADC was better than that of TRAb (Fig. 3, Tables III and IV). Sensitivity, specificity, accuracy, PPV and NPV were between 89 and $97 \%$ when the optimal cut-off value of $1.837 \times 10^{-3} \mathrm{~mm}^{2} / \mathrm{sec}$ was determined for ADC. TRAb has been demonstrated to be a useful serum marker to differentiate GD from PT, although false positives and false negatives remain $(2-3,15-19)$. The present study revealed that the sensi- 
tivity, specificity, accuracy, PPV and NPV were between 70 and $90 \%$ when the optimal cut-off value of $1.350 \mathrm{IU} / \mathrm{ml}$ was determined for TRAb. It is hypothesized that ADC in DWI reflects the cellular structure being more condense in PT than in GD due to massive lymphocyte infiltration with hyperplastic germinal center formation. Hypercellularity in PT results in more numerous structural components and membranes, resulting in greater impedance and restriction of the motion/diffusion of water molecules, which engenders low ADC values. Tezuka et al (23) conducted an investigation of 24 patients with GD, 5 patients with subacute thyroiditis and 5 patients with Hashimoto thyroiditis. They found that ADC values obtained from patients with GD were significantly higher than those from patients with subacute thyroiditis and Hashimoto thyroiditis. Tezuka et al (23) proposed another hypotheses, which was that perfusion due to augmented intrathyroidal blood flow and vascularity (characteristic of GD) might account for the significantly higher ADC values of GD, as compared with those of subacute thyroiditis and Hashimoto thyroiditis. It is also evident that, as in PT, lymphocytic infiltration is a prominent feature in subacute thyroiditis and Hashimoto thyroiditis (29), which will result in hypercellularity. This may explain why the results of the present study are consistent with those of Tezuka et al.

MR imaging is non-invasive, since no injection of contrast material is required. Another advantage is the lack of ionizing radiation, which is of particular relevance if patients are pregnant or nursing, or if a patient with recurrent disease requires repeated follow-up examinations. In addition, if the facility is not equipped to perform RAIU or thyroid scintigraphy, ADC could be a good option for imaging due to the broader availability of MR scanners. The MR examination time is longer than that of RAIU and is more uncomfortable for the patient. However for RAIU, the patient has to wait for $24 \mathrm{~h}$ prior to the measurement of thyroid radioactivity under the protocol used at Tianjin Medical University General Hospital. In addition, for thyroid scintigraphy, an uptake time of $\sim 30$ min must also be added. MR imaging, particularly DWI, has certain limitations. For instance, DWI is highly sensitive to motion artifacts, such as swallowing or breathing. Moreover, patients with pacemakers or incompatible metal implants cannot be examined by MR, although such cases were not encountered in the present study.

Several major limitations of the present study are acknowledged. First, the study population was small, so the results can only be considered to be preliminary. An extension of the study to a larger patient population is planned. Secondly, the number of cases of tissue sampling for histopathological evidence was limited, particularly for PT, where only 2 patients with several episodes of recurrence agreed to undergo a biopsy. It was not possible to conduct a statistical analysis of the pathological data, and no correlation was established between histopathology and ADC. Thirdly, image resolution improvement was required for ADC maps. Although no standard method for measuring ADC has been established, different methods of measuring ADC were not compared. Further evaluations that complement these limitations are necessary.

In conclusion, the present study showed that RAIU, ADC and TRAb are all of diagnostic value for reliably differentiating between GD and PT. Rationale for evaluating DWI to differentiate GD from PT were provided. ADC determined by DWI reflected tissue cellularity and intra-cellular and inter-cellular membranes, whereas changes in thyroid uptake were displayed by RAIU and thyroid scintigraphy.

\section{Acknowledgements}

This study was supported by Tianjin Medical University New Century Excellent Talent Program; Young and Middle-aged Innovative Talent Training Program from Tianjin Education Committee; and Talent Fostering Program (the 131 Project) from Tianjin Human Resources and Social Security Bureau (all awarded to Zhaowei Meng). This investigation was also supported by the National Key Clinical Specialty Project (awarded to the Departments of Nuclear Medicine and Radiology).

\section{References}

1. Bahn Chair RS, Burch HB, Cooper DS, et al; American Thyroid Association; American Association of Clinical Endocrinologists: Hyperthyroidism and other causes of thyrotoxicosis: management guidelines of the American Thyroid Association and American Association of Clinical Endocrinologists. Thyroid 21: 593-646, 2011.

2. Izumi Y, Takeoka K and Amino N: Usefulness of the 2nd generation assay for anti-TSH receptor antibodies to differentiate relapse of Graves' thyrotoxicosis from development of painless thyroiditis after antithyroid drug treatment for Graves' disease. Endocr J 52: 493-497, 2005.

3. Kamijo K, Murayama H, Uzu T, Togashi K and Kahaly GJ: A novel bioreporter assay for thyrotropin receptor antibodies using a chimeric thyrotropin receptor $(\mathrm{Mc} 4)$ is more useful in differentiation of Graves' disease from painless thyroiditis than conventional thyrotropin-stimulating antibody assay using porcine thyroid cells. Thyroid 20: 851-856, 2010.

4. Kubota S, Tamai H, Ohye H, et al: Transient hyperthyroidism after withdrawal of antithyroid drugs in patients with Graves disease. Endocr J 51: 213-217, 2004.

5. Iitaka M, Morgenthaler NG, Momotani N, et al: Stimulation of thyroid-stimulating hormone (TSH) receptor antibody production following painless thyroiditis. Clin Endocrinol (Oxf) 60: 49-53, 2004.

6. Misaki T, Miyamoto S, Kasagi K, Mori T and Konishi J: Serial occurrence of two types of postpartum thyroid disorders. Usefulness of Tc-99m pertechnetate uptake. Clin Nucl Med 21: 460-462, 1996.

7. Nagai Y, Toya T, Fukuoka K, et al: Occurrence and spontaneous remission of Graves' hyperthyroidism preceded by painless thyroiditis. Endocr J 44: 881-885, 1997.

8. Sarlis NJ, Brucker-Davis F, Swift JP, Tahara K and Kohn LD: Graves' disease following thyrotoxic painless thyroiditis. Analysis of antibody activities against the thyrotropin receptor in two cases. Thyroid 7: 829-836, 1997.

9. Umena S, Takano T, Iijima T, et al: A case of repeated painless thyroiditis followed by Graves' disease. Endocr J 42: 821-826, 1995.

10. Ho SC, Eng PH, Fok AC, Ng DC and Khoo DH: Thyrotoxicosis due to the simultaneous occurrence of silent thyroiditis and Graves' disease. Thyroid 9: 1127-1132, 1999.

11. Hiraiwa T, Ito M, Imagawa A, et al: High diagnostic value of a radioiodine uptake test with and without iodine restriction in Graves' disease and silent thyroiditis. Thyroid 14: 531-535, 2004.

12. Kidokoro-Kunii Y, Emoto N, Cho K and Oikawa S: Analysis of the factors associated with Tc-99m pertechnetate uptake in thyrotoxicosis and Graves' disease. J Nippon Med Sch 73: 10-17, 2006.

13. Osaki Y, Sakurai K, Arihara Z, Hata M and Fukazawa H: Prediction of late (24-h) radioactive iodine uptake using early (3-hour) uptake values in Japanese patients with Graves' disease. Endocr J 59: 173-177, 2012.

14. Morris LF, Waxman AD and Braunstein GD: Accuracy considerations when using early (four-or six-h) radioactive iodine uptake to predict twenty-four-hour values for radioactive iodine dosage in the treatment of Graves' disease. Thyroid 10: 779-787, 2000. 
15. Kamijo K: Study on cutoff value setting for differential diagnosis between Graves' disease and painless thyroiditis using the TRAb (Elecsys TRAb) measurement via the fully automated electrochemiluminescence immunoassay system. Endocr J 57: 895-902, 2010.

16. Yoshimura Noh J, Miyazaki N, Ito K, et al: Evaluation of a new rapid and fully automated electrochemiluminescence immunoassay for thyrotropin receptor autoantibodies. Thyroid 18: $1157-1164,2008$.

17. Ilicki A, Gamstedt A and Karlsson FA: Hyperthyroid Graves disease without detectable thyrotropin receptor antibodies. J Clin Endocrinol Metab 74: 1090-1094, 1992.

18. Costagliola S, Morgenthaler NG, Hoermann R, et al: Second generation assay for thyrotropin receptor antibodies has superior diagnostic sensitivity for Graves' disease. J Clin Endocrinol Metab 84: 90-97, 1999.

19. Morita T, Tamai H, Oshima A, et al: The occurrence of thyrotropin binding-inhibiting immunoglobulins and thyroid-stimulating antibodies in patients with silent thyroiditis. J Clin Endocrinol Metab 71: 1051-1055, 1990.

20. Amino N, Yabu Y, Miyai K, et al: Differentiation of thyrotoxicosis induced by thyroid destruction from Graves' disease. Lancet 2: 344-346, 1978

21. Izumi $Y$, Hidaka $Y$, Tada $H$, et al: Simple and practical parameters for differentiation between destruction-induced thyrotoxicosis and Graves' thyrotoxicosis. Clin Endocrinol (Oxf) 57: 51-58, 2002.

22. Ota H, Amino N, Morita S, et al: Quantitative measurement of thyroid blood flow for differentiation of painless thyroiditis from Graves' disease. Clin Endocrinol (Oxf) 67: 41-45, 2007.

23. Tezuka M, Murata Y, Ishida R, et al: MR imaging of the thyroid: correlation between apparent diffusion coefficient and thyroid gland scintigraphy. J Magn Reson Imaging 17: 163-169, 2003.

24. Zheng W, Jian T, Guizhi Z, Zhaowei M and Renfei W: Analysis of ${ }^{13}$ I therapy and correlation factors of Graves' disease patients: A 4-year retrospective study. Nucl Med Commun 33: 97-101, 2012.

25. Wang RF, Tan J, Zhang GZ, Meng ZW and Zheng W: A comparative study of influential factors correlating with early and late hypothyroidism after ${ }^{131} \mathrm{I}$ therapy for Graves' disease. Chin Med J (Engl) 123: 1528-1532, 2010.

26. Vitug AC and Goldman JM: Silent (painless) thyroiditis: Evidence of a geographic variation in frequency. Arch Intern Med 145 473-475, 1985

27. Meng Z, Zhu M and Tan J: Elephantiasis legs. Am J Med Sci 347: 248, 2014

28. Mittra ES and McDougall IR: Recurrent silent thyroiditis: A report of four patients and review of the literature. Tfhyroid 17: 671-675, 2007.

29. LiVolsi VA: The pathology of autoimmune thyroid disease: A review. Thyroid 4: 333-339, 1994.

30. Malayeri AA, El Khouli RH, Zaheer A, et al: Principles and applications of diffusion-weighted imaging in cancer detection, staging and treatment follow-up. Radiographics 31: 1773-1791, 2011.
31. Woodhams R, Ramadan S, Stanwell P, et al: Diffusion-weighted imaging of the breast: Principles and clinical applications. Radiographics 31: 1059-1084, 2011.

32. Padhani AR, Liu G, Koh DM, et al: Diffusion-weighted magnetic resonance imaging as a cancer biomarker: Consensus and recommendations. Neoplasia 11: 102-125, 2009.

33. Liu Z and Xiao X: The use of multi b values diffusion-weighted imaging in patients with acute stroke. Neuroradiology 55: 371-376, 2013.

34. Della Nave R, Foresti S, Tessa C, et al: ADC mapping of neurodegeneration in the brainstem and cerebellum of patients with progressive ataxias. Neuroimage 22: 698-705, 2004.

35. Pienaar R, Paldino MJ, Madan N, et al: A quantitative method for correlating observations of decreased apparent diffusion coefficient with elevated cerebral blood perfusion in newborns presenting cerebral ischemic insults. Neuroimage 63: 1510-1518, 2012.

36. Forbes KP, Pipe JG, Karis JP and Heiserman JE: Improved image quality and detection of acute cerebral infarction with PROPELLER diffusion-weighted MR imaging. Radiology 225: 551-555, 2002

37. Ahn SJ, Park MS, Kim KA, et al: ${ }^{18}$ F-FDG PET metabolic parameters and MRI perfusion and diffusion parameters in hepatocellular carcinoma: A preliminary study. PLoS One 8: e71571, 2013

38. Choi SH, Paeng JC, Sohn $\mathrm{CH}$, et al: Correlation of ${ }^{18} \mathrm{~F}-\mathrm{FDG}$ uptake with apparent diffusion coefficient ratio measured on standard and high $b$ value diffusion MRI in head and neck cancer. J Nucl Med 52: 1056-1062, 2011

39. Fruehwald-Pallamar J, Czerny C, Mayerhoefer ME, et al: Functional imaging in head and neck squamous cell carcinoma: Correlation of PET/CT and diffusion-weighted imaging at 3 Tesla. Eur J Nucl Med Mol Imaging 38: 1009-1019, 2011.

40. Nakajo M, Kajiya Y, Tani A, et al: FDG PET/CT and diffusion-weighted imaging of head and neck squamous cell carcinoma: Comparison of prognostic significance between primary tumor standardized uptake value and apparent diffusion coefficient. Clin Nucl Med 37: 475-480, 2012.

41. Ohno Y, Koyama H, Yoshikawa T, et al: N stage disease in patients with non-small cell lung cancer: Efficacy of quantitative and qualitative assessment with STIR turbo spin-echo imaging, diffusion-weighted MR imaging and fluorodeoxyglucose PET/CT. Radiology 261: 605-615, 2011.

42. Wu X, Pertovaara H, Korkola P, et al: Correlations between functional imaging markers derived from PET/CT and diffusion-weighted MRI in diffuse large B-cell lymphoma and follicular lymphoma. PLoS One 9: e84999, 2014.

43. Thoeny HC, De Keyzer F and King AD: Diffusion-weighted MR imaging in the head and neck. Radiology 263: 19-32, 2012.

44. Ho KC, Lin G, Wang JJ, et al: Correlation of apparent diffusion coefficients measured by $3 \mathrm{~T}$ diffusion-weighted MRI and SUV from FDG PET/CT in primary cervical cancer. Eur J Nucl Med Mol Imaging 36: 200-208, 2009. 